\title{
WHITE LEDS FOR RURAL LIGHTING
}

\author{
A.B.Sebitosi and ${ }^{+}$P. Pillay, Senior Member, IEEE \\ University of Cape Town, Dept of Electrical Engineering, Private Bag Rondebosch 7701. \\ ${ }^{+}$Clarkson University, Potsdam NY 13699
}

\begin{abstract}
This paper considers the concept of very advanced technologies being developed in the West, to solve rural electrification problems in the developing world. The myth that poor quality is cheaper and therefore more affordable for rural electrification is dispelled. On the contrary this paper points to instances when problems of the rural poor have been solved by using cutting edge technologies where standard solutions had proved unworkable.

In this paper a futuristic technology, the white light emitting diode (LED), for general lighting, is examined as a solution to African rural electrification. Comparisons are made with conventional technologies.
\end{abstract}

\section{INTRODUCTION}

Sub-Saharan Africa constitutes the bulk of humanity that is still not electrified. The national power utilities in those countries are struggling to cope with the existing infrastructure, which are actually shrinking, in real terms. Many go by acronyms like, 'No Electric Power Again', to stand for 'National Electric Power Authority'! As a result the current trend is for communities to fend for themselves. Small remote power installations, especially from renewable energy are characterized by limited generation and storage capacities. These resource constraints call for effective demand side management that conserves energy but with minimal compromise on service delivery quality.

In these communities, lighting is dominated by smoky kerosene candles and is responsible for perennial respiratory and sight illnesses, in addition to the missed learning, business and other opportunities. This makes lighting a priority activity in rural electrification. Globally, electric lighting accounts for some one fifth of all electric energy consumed, and a similar percentage of electric energy related green house gas (GHG) emissions. Therefore any efficient electric lighting initiative is a subject of interest for all humankind.

Lighting loads are often inaccurately modeled due to omission of a number of relevant non-electrical parameters. Illumination as a technology has made its own advances, independently, that must be included in the ultimate electrical lighting solution [1]. High benefit lighting in the work place for example, optimizes sight dependent tasks while minimizing the energy used. Illumination experts [8] point out that over-lighting a space or task area degrades the lighting quality in addition to wasting energy.

Solutions for electrification or energy development in the developing world need not follow the same path as for the developed world. Instead relevant technical solutions for advanced applications in the developed world can be used to leapfrog intermediate technologies and applied directly, with benefit to the developing countries. One example is the use of cell phones, which do not require the costly physical infrastructure of regular land phones. Very remote rural locations that would have otherwise not dreamt of getting any communication are now just a push button away from the rest of the world.

In the field of lighting, recent developments in automotive electronics may yet launch another cutting edge technology into the rural communities. The red light emitting diode (LED) in the traffic and car tail light industries, in North America, reported a market penetration of $20 \%$, rising from a mere 8\% during an eighteen month period leading up to 2002. Energy savings of up to $80 \%$ are reported. This LED is fast evolving into the white LED for general lighting and may be applied directly to rural electrification problems.

This paper examines the lighting issue at depth. Section 2 has a description of how radiometric power output of a light source (in watts) relates to photometric or light power (in lumens) by incorporating the human eye frequency response. A range of attributes of a light source is described. How they contribute to the lighting quality and thus finally impacting on the electric power source is explained. Section 3 compares the energy consumption and cost, in a rural home, of incandescent lights vs LED light sources. Conclusions are then drawn on the possible impact of LEDs on rural electrification.

\section{BACKGROUND: LIGHTING LOAD MODELING}

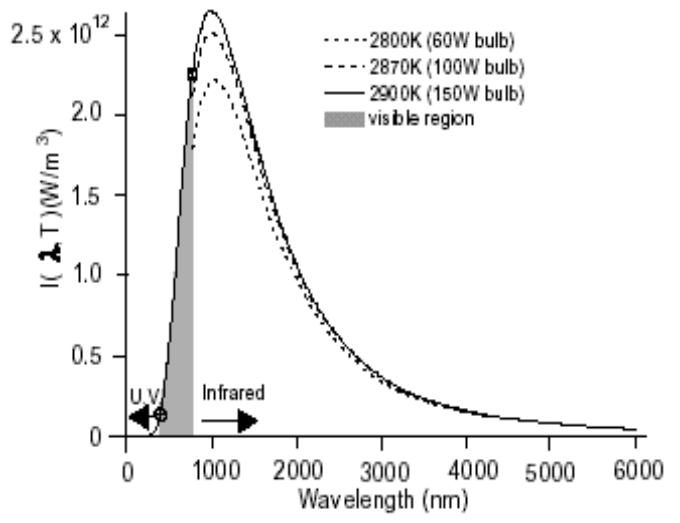

A.

Figure 1: Output energy as a function of wavelength for an incandescent bulb 
When connected across a voltage source, an incandescent light The following is an elementally illustration of a general bulb draws a current with a waveform that is a near replica of monochromatic case. The symbols used are not the authentic (and in phase with) the voltage and therefore at a near unity illumination symbols but merely serve to illustrate a point.

power factor. This is the classic criterion for an ideal electric

load, yet this, apparently, ideal load is labeled as inefficient: in Let $y_{1}=f_{1}(\lambda)$ watts, represent the radiometric function fact, very inefficient.

Modeling lighting loads is unique as it involves the human eye as the ultimate load determining the required electric power input. Any losses prior to the eye constitute power delivery losses, and failure by light to reflect off an intended target and then to the reception of a normal eye constitutes system inefficiency.

In figure 1, the curve represents the radiometric output power, in watts, of a tungsten incandescent light source as a function of wavelength. The shaded area represents the visible output of the source, defined as the range from 360 nanometers (nm) to $830 \mathrm{~nm}$. This fraction determines the efficacy of the light source. However, since the human eye responds differently to different wavelengths (within the visible range), the light source efficacy is not (literally) the shaded area divided by the total area. In the above example with fog, a mere shift to a yellowish/orange headlamp may improve visibility and remove the necessity to increase headlamp power rating.

Figure 2 is an empirical curve, drawn by the International Commission on Illumination (CIE), of an average human eye response as a function of wavelength. This is the photopic curve. (The scotopic curve is the response during very low light levels and is not part of this illustration.).

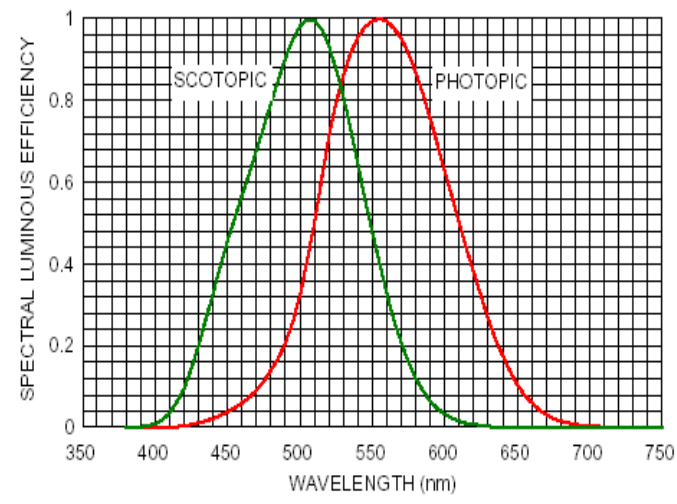

\section{LIGHT SOURCE PERFORMANCE}

As mentioned, efficacy is given as the number of lumens of light output of a light source per input watt of electrical power $(\mathrm{lm} / \mathrm{w})$. This is, however, just one of the indices of lighting quality. For a more comprehensive light source design one requires more data. Initially the task for which light is required must be defined and the required amount of light quantified.

As an example one may consider an incandescent light source and a light emitting diode (LED), which currently (2002) have practically the same lumens per watt (efficacies) rating. It would therefore be reasonable to assume that the two light sources would consume the same amount of energy to perform the same task. How then could the traffic and signal lights industry report such massive energy savings of up to $80 \%$ by just replacing the incandescent lights with LEDs?

As seen on the photopic function the human eye responds best at $555 \mathrm{~nm}$ This point defines the full coefficient, 1 , of the eye's response. The unit of light power is the lumen $(\mathrm{lm})$. The eye receives radiometric watts and interprets them in lumens using the response function. For example, 1 watt of (monochromatic) radiometric power at $500 \mathrm{~nm}$ will be interpreted, in lumens, as 0.3 in value compared to 1 watt at $555 \mathrm{~nm}$. (See figure 2)

In the case of a 'stop' traffic light, for example, the required task is the production of red light and only to the view of the car driver. The key words are color and directivity. True efficiency must therefore be the amount of power successfully converted for the task per unit watt of input of electric power. 
In order to perform this task the incandescent light must use a reflector and a red filter. A 140 watt incandescent lamp with an efficacy of 15 lumens per watt will produce 2100 lumens but after the red filtering and reflector the amount of red light that is finally available to the driver may be only 200 lumens, which happens to be adequate. A red LED, on the other hand, is a monochromatic device and has directivity with an appropriate angle. It is task specific: requiring neither filter nor reflector. Therefore a replacement LED assembly for the same traffic light function only requires 200 lumens. Having the same efficacy as the incandescent, the LED ends up consuming only $10 \%$ of the power. Moreover the LED has other superior attributes like shock resistance; a problem that causes premature failure in incandescent traffic lights and vehicle and tail lights.

Light sources have a variety of other attributes in varying degrees as illustrated in the appendix. These determine the suitability and therefore efficiency of a light source for a given task. In addition the color of the surroundings having unique reflective properties which will affect the amount of light required and ultimately impact on the required generation and/or storage capacity in an electrification design. "In some cases enhancement of these influencing factors can improve performance without the need to raise illuminance" [2].

As mentioned earlier in the case of traffic lights, confining light to a specific purpose does improve on energy efficiency. The effectiveness of this technique, called 'tasking', has also been demonstrated by Philips engineers. A set of specially constructed LED street lights performed at par with sodium lamps despite the overwhelmingly superior efficacy of the sodium.

If the purpose for a light source is reading it would appear reasonable to infer that energy would be most conserved if the light is confined to a target area, namely the book. Illumination experts, however, caution that this may cause discomfort due to glare [4], if the background is pitch dark. Other symptoms include annoyance and reduced productivity. Some mild ambient lighting, of the order of at least 10\%, which would otherwise be inadequate on its own, is recommended [8].

Finally there are special circumstances that call for higher lamp lumen levels than would be required ordinarily. These include provision for visually impaired persons, or special age groups of occupants.

\section{SOLID-STATE Lighting (SSL)}

SSL has taken a foothold and is certain to revolutionalise lighting energy consumption. Cynics refer to traditional electrical lighting as a process of heating of a medium by the application of electricity until it's hot enough to give out some light. A grossly energy-squandering scenario!
In contrast LED technology involves a quantum process to convert dc current to light. Conversion efficiencies nearing $100 \%$ have been achieved in laboratory results with certain materials. However it is the extractability of this light that is still one of the stumbling blocks In fact it appears as though the materials with the most efficient quantum conversions have the poorer extractability efficiencies. This is part of what research will address and should be awaited for with much anticipation by the African rural communities. Historically the LED efficacy has been doubling every 18 to 24 months since the beginning of the 1970's. Such evolution is highly dependent on funding. In July 2001 the US Senate launched, 'the Next Generation lighting Initiative' with an ambitious goal to achieve 25\% market penetration by the year 2012 . Subsequently a bill was passed in April 2002, committing over a billion dollars per year for research and development (R\&D) for the next generation of lighting technologies.

LEDs have superior life spans to incandescent light sources. However, like other light source types they suffer from lumen depreciation and one has to exercise caution with hyped figures like 100,000 hours. This gradual deterioration, once understood, could be factored into scheduled maintenance. Unlike the abrupt failures by incandescent lamps it would be an added advantage.

The time it takes to start a light source can in certain instances be important. Currently, CFLs are very popular in off grid systems. There is a time delay to get to full brightness. So the incandescent lamp still ends up standing in for that odd function like closet lighting. The LEDs are not only as fast as the incandescent lights to start but even faster when going off.

\section{LIGHTING REQUIREMENTS OF A RURAL HOME}

In Kenya, as in most sub- Saharan countries, the overwhelming majority of the populations live in the rural areas with official grid electrification close to $1 \%$ or virtually non-existent. Current privatization trends of public corporations have led to further divesture from the rural areas and dashing any hopes for further grid extensions. As a result recent developments have been mainly driven by self-help initiatives. The available means to harness the all-vital electric energy are small and costly. All efforts must be made to economize the meager resources without compromising quality of service.

As mentioned earlier a light source may produce a large amount of light and with a good electricity to light conversion efficiency (efficacy) but how well it targets a particular the intended task will impact on its overall efficiency. Currently (2002) the efficacies of incandescent lamps and white LEDs are of the same order.

Household lighting applications are in three general categories. Ambient lighting is a basic minimum amount of illumination for living. This level should be adequate for 
sitting rooms, corridors and for more public utilities like restaurants and hospital wards. General lighting provides a relatively higher illumination level than ambient. It is for such purposes as reading, cooking and security. Task lighting is the illumination required for very detailed viewing, like an operating theater.

Regulations, guide lines and on occasions legislation specify these illumination requirements in lumens per flat surface area. In SI units 1 lumen per square meter is a lux.

In South Africa, a code of practice [8] gives the minimum lux for a whole range of locations and activities. For example, kitchens are allocated 200 lux, 100 lux for bathrooms and 500 for study and reading. Like other standards, there may be variations from country to country. In the following illustration the specification of the South African Bureau of Standards will be assumed.

An incandescent lamp and a white LED for reading are compared below. If the reading area is $0.25 \mathrm{~m}^{2}$ and the required light density is 500 lumens per square meter, then the lamp should give $(\mathbf{5 0 0} \times \mathbf{0 . 2 5})=\mathbf{1 2 5}$ lumens.

As of 14th April 2002 Lumileds produced LUXEON-5W, a 120 lumen white LED light source with a power consumption of 5 watts. As mentioned earlier, LEDs have directivity and it is reasonable to assume that all the light can be confined to the required area.

The incandescent lamp will use a reflector (luminaire) to attain the directivity. A good quality luminaire has a coefficient of utilization (CU) of 0.55 [8]. $\mathrm{CU}$ is the indication of the proportion of useful light emitted by a luminaire. Therefore in order to create the same effect as the Luxeon-5 the incandescent light must produce 120/0.55 $=218$ lumens. The efficacy of a typical incandescent lamp is 15 lumens per watt. The wattage of this lamp will be $218 / 15=$ 14.5 watt. Incandescent lamp efficacies rise with filament temperature, which in turn rises with lamp wattage. So in reality a lamp of such low wattage will have an efficacy closer to 10 lumens per watt. This will make its wattage closer to 20 watts and hence will require 4 times the amount of power as the Luxeon-5.

\section{1) 6. The Future}

As of 2002 the commercial white LED has reached an efficacy mark of $25 \mathrm{~lm} / \mathrm{w}$. According to Sandia National Laboratories the projected efficacy for the white LED before the end of this decade is 50 lumens per watt with a possibility of nearing 100 with accelerated effort $[12,13]$. According to the projection model in [12] the accelerated track option is conditional upon adequate funding.

On the $25^{\text {th }}$ of April, 2002 the US Senate passed bill S.517, and committed over 1 billion US dollars per annum for research and development (R\&D), for "such areas as next generation lighting technologies." This is cause for optimism. The same forecast projects that at 50 lumens per watt the per kilo lumen price will still be US\$ 8.30 against the incandescent lamp's current US\$ 0.56. Cost is by far the major disadvantage of the LED. If the accelerated track should be followed a price breakthrough of US\$ 0.50 is projected at $120 \mathrm{~lm} / \mathrm{w}$ of component efficacy.

It should be noted that the Sandia model focused on efficacy as the major index for market response. There are other possibilities. The market could, for example, be impressed by other improving attributes like, the life span or color rendering index CRI and realize a price break through much earlier.

Life spans of incandescent lamps average about 800 hours. Independent researchers have pegged the $50 \%$ lumen depreciation life span of a white LED to a more realistic figure of 6000 hours $[10,11]$. This is a far cry from the manufacturers' frequent claims of 100,000 hours, which is probably when the LED finally shuts down. It is nonetheless far superior to the incandescent lamp and matches the CFLs, while at the same time not being completely out. (CFLs have additional shortcomings like disposal.) This gives an 8:1 advantage of cost over life span of the white LED against the incandescent lamp. It means that for every time one buys an LED lamp one requires to buy at least 8 incandescent lamps to last as long, giving a much better cost effectiveness scenario in favor of the LED. It also means that even at the projected lower end value of $50 \mathrm{~lm} / \mathrm{w}$ the LED will be far more cost effective to buy than the incandescent lamp while LED power consumption will be less than a third.

At the current levels of technology the white LED's advantage over the incandescent lamp would appear to be mainly in task applications, like reading and perhaps the kitchen. Additionally when considering lumen depreciation (also called light loss factor, LLF), the LED scores yet another point. Because the reflectance of the reflector deteriorates as well, the incandescent lamp has two depreciation factors to contend with against the LED's one. Otherwise with virtually the same efficacies elsewhere in the rural household, power consumption levels are matched.

Consider a rural African household comprised of two bedrooms, a kitchen, a bath room with a toilet and a lounge/dinning at the far end. One may assume over $80 \%$ of the full lighting load for the four hours per evening and only incandescent lamps in use. Assuming also, that the room sizes and surrounding wall reflectances are such that specified lux levels can be achieved using, $60 \mathrm{~W}$ in the kitchen, $40 \mathrm{~W}$ in each of the bedrooms $25 \mathrm{~W}$ in the bathroom, $60 \mathrm{~W}$ for ambience in the lounge/dinning room and an additional $20 \mathrm{~W}$ for the table reading lamp. The total peak load would be $225 \mathrm{~W}$. At the assumed load factor of $80 \%$ for the period of usage the average rating would be $180 \mathrm{w}$. During a 4 hour evening a total of 720 watt hours $(\mathrm{Wh})$ would be consumed. With a simple automobile lead acid battery system that permits only a $40 \%$ depth of discharge (DOD) one would require a storage capacity of $720 / 40 \%=1800 \mathrm{Wh}$. 
Under the current circumstances replacing the incandescent lamps with white LED's would only affect the reading light and reduce the peak load by $15 \mathrm{~W}$. Given that batteries are only available in certain sizes, it should be reasonable to assume that there would be no difference on the storage capacity.

If one should consider the same LEDs having evolved to efficacy levels of $120 \mathrm{~lm} / \mathrm{w}$ then the average load would become (180 $\mathrm{x}$ incandescent efficacy)/(LED efficacy)

Average load $=(180 \times 15) / 120=22.5$ watts

This would yield a required storage of $90 \mathrm{Wh}$ and a lead acid battery size of $225 \mathrm{Wh}$. By that time the life span could have possibly exceeded the 100,000-hour mark and the CRI perhaps at 100 .

\section{CONCLUSION}

This paper has examined the concept of applying first world innovative technologies to the problem of rural electrification. In particular, white LEDs can electrify rural Africa, with minimal power requirements.

\section{APPENDIX}

Color rendering index $(\mathrm{CRI})$ is a relative scale from $0-100$ and determines how colors of an object are perceived under an artificial light as compared to a reference light. The lowpressure sodium lamp, for example, while reputed to have the highest efficacy figures of the order of 130 to 150 lumens/watt, has a CRI of nearly zero. So it can only be said to be efficient for the outdoors and certainly very inefficient indoors, as most colors appear black under this light. The incandescent lamp, on the other hand has a very high CRI of nearly 100 . Or put simply, colors appear most agreeable under light from an incandescent bulb. This could partly explain why even people who are clearly aware of relative lamp performances, and energy benefits and can afford the 'better' types (like CFLs) still keep choosing incandescent lamps. The current white LEDs perform very well with a CRI of 85, which matches the compact fluorescents, (CFLs) and they will get better. Besides, CFLs use toxic mercury to function and their disposal is a problem.

Lumen depreciation is the reduction in output lumens of a light source with age. The depreciation rate is dependent on lamp type, environment and rate of usage. The life span of a light source is deemed to have ended if the amount of lumens falls below $50 \%$ of the initial figure. "In lighting design we must take into account this fall by use of a maintenance factor"'[3]. This is one piece of data that is frequently a subject of distortion by manufacturers. The fact that lumens per watt values on the labels are only valid for new lamps is hardly ever mentioned. However considering this omission as a constant across the board, LEDs have relatively far longer life spans than any other type.

\section{REFERENCES}

[1] "Lighting Education (1983 - 1989)," Publication CIE 99 1992. ISBN 3900734364

[2] "Lighting of indoor work places," Publication CIE Standard: S008/E - 2001

[3] "Maintenance of indoor electric lighting systems," Publication CIE 97 - 1992 ISBN 3900734348

[4] "Discomfort glare in interior lighting," Publication CIE 117 - 1995 ISBN 3900734704

[5] "Proceedings of the first CIE symposium on lighting quality" $\mathrm{C}$ 1998 ISBN 3900734917

[6] William H. Casting, "Distribution system modeling and analysis" CRC Press LLC, 2002

[7] Glover, J. D. and Sarma, M., "Power system analysis and design, $2^{\text {nd }}$ edition, PWS-Kent Publishing, Boston, 1995.

[8] SABS 0114-1:1996. Interior Lighting. (Part 1: Artificial lighting of interiors). South African Bureau of Standards [9] SABS 0114-2:2002. Interior Lighting. (Part 2: Emergency lighting). South African Bureau of Standards.

[10] N Narendran et al. 2000, "Characterizing white LEDs for general illumination applications." Proc. SPIE 3950. [11] N Narendran et al. 2001a, "What is useful life for white light LEDs?” J. Illum. Eng. Soc. 30(1) 57.

[12] T. Drennen, R. Haitz, J. Tsao, "A market diffusion and energy impact model for solid-state lighting. SAND2001 2830." Sandia National Laboratories, Agilent Technologies, E20 Communications and Hobar \& William Smith Colleges. [13] Arthur D. Little, Inc, "Energy saving potential of solid state lighting in general light applications". US Department of energy. 